\title{
Téoros
}

Revue de recherche en tourisme

\section{Le tourisme durable, un enjeu de rééquilibrage territorial dans le département français des Pyrénées-Orientales}

\section{Nicolas Berthet}

Numéro hors-série, 2012

Innovations en tourisme durable

Innovations in Sustainable Tourism

URI : https://id.erudit.org/iderudit/1036562ar

DOI : https://doi.org/10.7202/1036562ar

Aller au sommaire du numéro

Éditeur(s)

Université du Québec à Montréal

ISSN

0712-8657 (imprimé)

1923-2705 (numérique)

Découvrir la revue

Citer cet article

Berthet, N. (2012). Le tourisme durable, un enjeu de rééquilibrage territorial dans le département français des Pyrénées-Orientales. Téoros, 49-54. https://doi.org/10.7202/1036562ar

\section{Résumé de l'article}

Adossé à la frontière espagnole, entre Méditerranée et Pyrénées, le département français des Pyrénées-Orientales, aussi nommé Pays Catalan, Catalogne Nord ou Catalogne française, est longtemps apparu comme une périphérie rurale, éloignée et faiblement industrialisée. La mise en place à partir des années 1960 de la Mission Interministérielle d’Aménagement touristique du littoral du Languedoc-Roussillon (aussi appelée Mission Racine, du nom de son dirigeant Pierre Racine), allait cependant rapidement convertir ce département en une destination touristique majeure du sud de la France. La dynamique de développement de la plaine littorale contrastait alors avec l'arrière-pays de la déprise agricole et de l'exode rural. Conscientes du potentiel touristique qu'offrait néanmoins un cadre naturel rural et préservé, diverses populations néorurales s'installèrent dès les années 1970 dans l'arrière-pays. Elles y développeront au tournant des années 1980 le tourisme de nature. Bien que marginal par rapport à l'activité générée par le tourisme de masse du littoral et des stations de ski, le tourisme de nature de l'arrière-pays constituait le point de départ d'un futur développement durable du tourisme dans les Pyrénées-Orientales.

À partir des initiatives de mise en réseaux des parties prenantes et des politiques menées en matière de transport public, cet article propose de décrire des processus d'innovation mis en place et/ou soutenus par le Conseil Général des Pyrénées-Orientales en matière de tourisme durable. Fruit de discussions engagées dans le cadre professionnel avec divers acteurs du tourisme local, cet article souligne l'importance que revêt l'engagement des collectivités locales, en l'occurrence le Conseil Général des Pyrénées-Orientales, pour mener à bien le développement durable du tourisme au sein d'une destination méditerranéenne encore largement dévolue au tourisme de masse.
Ce document est protégé par la loi sur le droit d'auteur. L'utilisation des services d’Érudit (y compris la reproduction) est assujettie à sa politique d'utilisation que vous pouvez consulter en ligne.

https://apropos.erudit.org/fr/usagers/politique-dutilisation/ 


\title{
Le tourisme durable, un enjeu de rééquilibrage territorial dans le département français des Pyrénées-Orientales
}

\author{
Nicolas BERTHET \\ Doctorant \\ Faculté de tourisme, Université de Gérone (Catalogne) \\ Université de Perpignan (France) \\ nmberthet@gmail.com
}

RÉSUMÉ: Adossé à la frontière espagnole, entre Méditerranée et Pyrénées, le département français des PyrénéesOrientales, aussi nommé Pays Catalan, Catalogne Nord ou Catalogne française, est longtemps apparu comme une périphérie rurale, éloignée et faiblement industrialisée. La mise en place à partir des années 1960 de la Mission Interministérielle d'Aménagement touristique du littoral du Languedoc-Roussillon (aussi appelée Mission Racine, du nom de son dirigeant Pierre Racine), allait cependant rapidement convertir ce département en une destination touristique majeure du sud de la France. La dynamique de développement de la plaine littorale contrastait alors avec l'arrière-pays de la déprise agricole et de l'exode rural. Conscientes du potentiel touristique qu'offrait néanmoins un cadre naturel rural et préservé, diverses populations néorurales s'installèrent dès les années 1970 dans l'arrière-pays. Elles y développeront au tournant des années 1980 le tourisme de nature. Bien que marginal par rapport à l'activité générée par le tourisme de masse du littoral et des stations de ski, le tourisme de nature de l'arrière-pays constituait le point de départ d'un futur développement durable du tourisme dans les Pyrénées-Orientales.

À partir des initiatives de mise en réseaux des parties prenantes et des politiques menées en matière de transport public, cet article propose de décrire des processus d'innovation mis en place et/ou soutenus par le Conseil Général des PyrénéesOrientales en matière de tourisme durable. Fruit de discussions engagées dans le cadre professionnel avec divers acteurs du tourisme local, cet article souligne l'importance que revêt l'engagement des collectivités locales, en l'occurrence le Conseil Général des Pyrénées-Orientales, pour mener à bien le développement durable du tourisme au sein d'une destination méditerranéenne encore largement dévolue au tourisme de masse.

Mots-clés: destination, développement durable, patrimoine, partie prenante, transport.

Depuis plus de trente ans, le concept de développement durable du tourisme constitue un centre d'intérêt majeur et communément partagé entre théoriciens et professionnels du tourisme (Sharpley et Telfer, 2000). Si l'idée de développer un tourisme intégré et intégrateur des territoires et des humains est acceptée par tous, la nature même du concept divise les communautés de chercheurs et de professionnels en deux catégories : les «tourism-centric» (Hunter, 1995) qui défendent l'idée que le tourisme durable constitue en soi une activité économique, et les autres qui l'envisagent davantage comme un simple élément des politiques de développement durable en général (Cronin, 1990).

Au-delà de ces débats sur la nature du développement du tourisme durable, l'Union européenne (2007), première destination touristique mondiale, s'est dotée de son propre Agenda 21 du tourisme dont les objectifs sont :

- la prospérité économique;

- l'équité et cohésion sociale; et

- la protection de l'environnement et de la culture.
La poursuite de ces objectifs au sein de destinations dévolues au tourisme de masse depuis cinquante ans, suppose la mise en place dans les départements de politiques volontaristes impulsées par les collectivités territoriales en général (Conseil Général, Communauté d'Agglomération, de communes, communes) et les Conseils Généraux en particulier, en partenariat avec l'ensemble des groupes de parties prenantes.

De l'écoute des attentes et ressentis de ces derniers en matière de tourisme dépend leur bonne implication (Hunter, 1997) au processus d'élaboration des politiques (Hardy et Beeton, 2001) et la prévention de tout conflit lié aux intérêts mis en jeu (Gee et Fayos-Sola, 1997). Le degré d'implication des parties prenantes conditionne leur influence dans l'élaboration des politiques de développement touristique et les avantages qu'elles pourraient en retirer sachant que cette participation communautaire reste cruciale pour le développement durable du tourisme (Campbell 1999, 2002; Murphy, 1988; Jones, 2005). 
Acteurs institutionnels de la vie économique, sociale et culturelle de proximité, les collectivités territoriales constituent un levier essentiel du développement du tourisme durable dans les villes, départements et régions. Affranchies de toute contrainte de rentabilité financière immédiate, elles initient plus librement des pratiques innovantes qui accompagnent et soutiennent le renouvellement des modèles de développement des destinations touristiques dans le cadre d'un aménagement raisonné des territoires.

Plus sensible que n'importe quel autre partie prenante à la stabilité financière, à la rémunération et à la viabilité économique, le secteur privé conditionne lui son implication dans le développement du tourisme durable au sein d'une destination au marché potentiel qu'il peut générer à partir de ressources naturelles et historiques préservées (Simpson, 2008). Afin de réussir leur intégration dans la dynamique touristique d'une destination, les parties prenantes privées entretiennent de bonnes relations avec les autres communautés impliquées ou touchées par le développement durable du tourisme (Beeton, 2006; Hawkins et Mann, 2007; UNWTO, 2005; Wearing et McDonald, 2002).

Pierre angulaire du développement du tourisme durable autour de la tradition et du patrimoine, l'identité territoriale revêt une importance de premier ordre dans la construction et la promotion d'une destination touristique. Aussi, l'implication de l'ensemble des parties prenantes est capitale puisqu'elle participe à l'identification du produit touristique à travers les spécificités locales dont chaque groupe d'acteurs est porteur, animateur et ambassadeur. Les acteurs institutionnels du tourisme tant au niveau national, régional que local, orientent d'ailleurs l'essentiel de leurs actions de communication vers la présentation et la promotion de l'identité territoriale dans le but d'attirer davantage de touristes tout en assurant un partage élargi du marché (Dredge et Jenkins, 2003).

Au niveau local, l'activité touristique apparaît souvent comme divisée, fragmentée et dispersée dans l'espace, générant une multitude d'initiatives économiques individualisées ne répondant à aucune logique de complémentarité au sein d'un réseau global d'acteurs. La dimension identitaire d'une destination peut contribuer à rassembler les différents groupes de parties prenantes autour de sentiments d'appartenance ou de passions communément partagés. Cela conduit naturellement les institutions du tourisme à construire leurs politiques d'harmonisation de l'espace autour de la territorialisation des identités culturelles locales (Pearce, 1992), à partir du patrimoine historique et naturel local.

$\mathrm{Si}$ innover consiste à introduire de nouvelles pratiques ou idées dans un processus commun d'action, dans le cadre du tourisme durable, l'innovation peut se construire à partir du patrimoine historico-culturel d'une destination en récupérant l'identité comme lien entre passé, présent et futur envisagés à partir de pratiques sociales répondant à un modèle de développement économique durable.

Parmi ces pratiques sociales, l'adoption des transports en commun pour découvrir les diversités culturelles d'un territoire représente un vrai progrès en termes de développement durable. La mise en place d'un système de transport en commun efficient permet l'accès aux ressources patrimoniales, en augmentant le nombre de visiteurs, tout en limitant le trafic automobile et les émissions de gaz polluants. Le budget alloué par les touristes au transport, à l'hébergement et à la vie sur leur lieu de vacances étant lié à leurs préférences et à une question de coûts, la question de la commodité des transports et de leur prix conditionne grandement le choix d'une destination touristique (Prideaux, 2000).

Nous verrons dans cet article, comment l'ensemble de ces problématiques conditionne l'élaboration des politiques de développement durable du tourisme au sein des PyrénéesOrientales, une destination touristique encore largement dévolue au tourisme de masse.

\section{Méthodologie}

La lecture de brochures d'informations éditées par les organismes impliqués dans le tourisme ainsi que la consultation des sites Internet de ceux-ci, ont permis de compléter les réflexions et connaissances partagées au cours de discussions engagées avec plusieurs acteurs du tourisme des PyrénéesOrientales dans le cadre professionnel.

Concernant les données relatives aux statistiques de fréquentation du «Bus à 1 euro» dont il sera question dans cette étude, des contacts par courriel et par téléphone ainsi qu'une rencontre avec la responsable du pôle voyageurs à la Direction des Déplacements et de l'éco-mobilité du Conseil Général des Pyrénées-Orientales n'ont permis d'obtenir que quelques données chiffrées fiables étant donné que certaines n'étaient toujours pas disponibles, ou inexistantes (statistique de fréquentation aux arrêts) ou bien encore inaccessibles car confidentielles. Les chiffres disponibles concernaient la fréquentation globale de chaque ligne, depuis Perpignan jusqu'à son terminus. Si le succès d'un réseau de transport en commun très bon marché le «Bus à 1 euro » est acquis en termes de fréquentation globale annuelle, il est encore difficile de mener une enquête qualitative de l'impact de cette politique sur chaque ligne et chaque commune desservie à partir des données disponibles.

Les informations relatives aux politiques d'entretien, de valorisation et de promotion du patrimoine historique et naturel, ont été obtenues par la consultation des sites internet et des brochures de promotion des organismes institutionnels du tourisme local (Offices de Tourisme, Comité départemental du tourisme, associations et fédérations travaillant pour les institutionnels).

\section{Les expériences d'innovation en matière de tourisme durable dans les Pyrénées-Orientales}

Adossé à la frontière espagnole, dans la Région LanguedocRoussillon, le département des Pyrénées-Orientales, compte 454737 habitants (INSEE, 2012) pour une superficie de $4116 \mathrm{~km}^{2}$ (voir illustration 1). Depuis le littoral méditerranéen jusqu'aux neiges des sommets pyrénéens, une grande variété de paysages à l'attrait naturellement renforcé par un riche patrimoine historique déclinent une géographie avantageuse et une historie riche. Ces atouts contribuent à faire de ce territoire le $7^{\mathrm{e}}$ département touristique français avec plus de 33 millions de nuitées (ODT Pyrénées orientales, 2011). 
Illustration 1 : Présentation et situation du département des Pyrénées-Orientales (source: Gildas Girodeau pour cette étude).

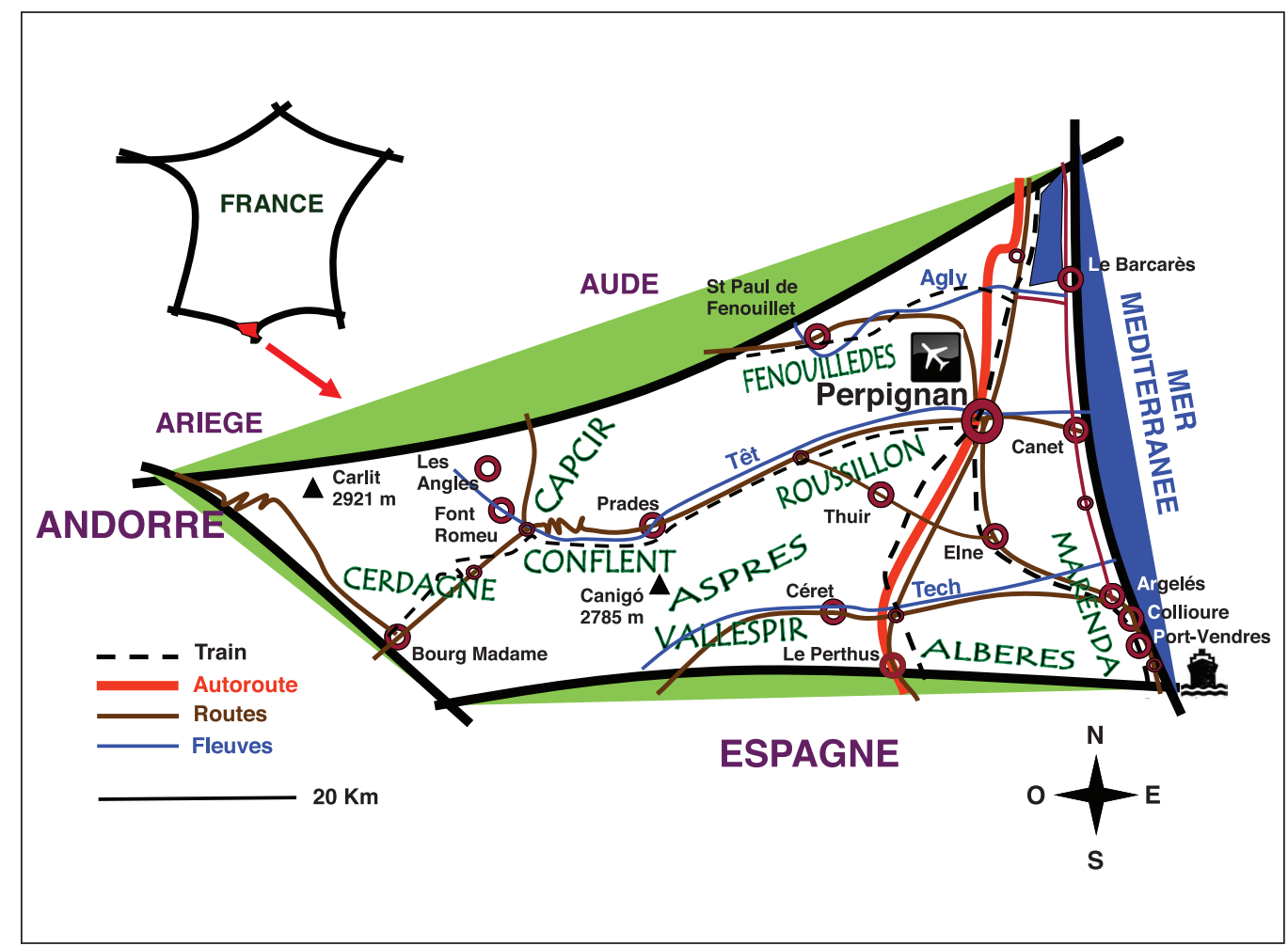

La survie et la revitalisation des périphéries rurales passent de nos jours par la conduite de politiques économiques et sociales de cohésion spatiale afin d'assurer une compétitivité plus équilibrée des territoires. Comme le soulignait Léon Bertrand (2005), ancien Ministre français délégué au tourisme, "dans une grande variété de situations, le tourisme offre l'opportunité de créer de la valeur ajoutée sur chaque territoire rural, tout en contribuant à en sauvegarder les richesses environnementales et à en conforter l'assise sociale, selon les principes du développement durable». Dans les Pyrénées-Orientales trois actions importantes menée et soutenues par le Conseil Général participe directement au développement du tourisme durable à l'échelle départementale, à savoir : 1) l'optimisation de la redistribution des bénéficies liés à l'activité touristique en fédérant les parties prenantes; 2) l'encouragement à la mobilité en facilitant l'accès aux différents sites touristiques du département par le biais d'un tarif unique de un euro pour tout déplacement effectué avec les bus du Conseil Général; 3) le positionnement de la coopération transfrontalière au cœur du développement durable du tourisme.

\section{Optimiser la redistribution des bénéficies liés à} l'activité touristique en fédérant les parties prenantes C'est dans les années 1990 que fût créée l'association Réseau Culturel (www.reseauculturel.fr) avec pour ambition de remettre le patrimoine départemental du pays catalan au cœur des dynamiques de développement touristique des Pyrénées-Orientales. En fédérant les sites patrimoniaux, en assurant la mise en réseau des acteurs et en développant la formation des techniciens du tourisme, Réseau Culturel Terres Catalanes a déjà permis de donner plus de visibilité au produit culturel unique que constitue l'ensemble du patrimoine local notamment grâce à l'édition chaque année du guide «Pass Intersites » qui offre des réductions à l'entrée des différents sites affiliés au Réseau Culturel.

En impliquant chaque année un peu plus les artisans, agriculteurs, hébergeurs et restaurateurs dans le processus de mise en valeur des traditions et savoirs faires, la fédération «Tourisme de Terroir» (www.tourismedeterroir.fr) participe à la promotion touristique des Pyrénées-Orientales à travers leurs produits naturels et gastronomiques. Du mois de mars au mois de septembre, l'organisation d'apéritifs de terroir itinérants permet à de nombreux professionnels d'aller à la rencontre des différentes clientèles touristiques, tout en assurant auprès de celles-ci la promotion des différents terroirs qui composent le département.

\section{Encourager la mobilité en facilitant l'accès} aux différents sites touristiques du département :

\section{le «Bus à 1 euro »}

Développer un modèle de tourisme durable dans un département frontalier de l'Espagne comme les Pyrénées-Orientales, suppose de composer avec un trafic automobile extrêmement dense en haute saison touristique. Pour limiter l'impact des transports sur l'environnement et assurer une meilleure accessibilité aux ressources patrimoniales du département, une politique ambitieuse et audacieuse en matière de transports en commun fut envisagée, décidée, planifiée puis mise en place par le Conseil Général des Pyrénées-Orientales. 

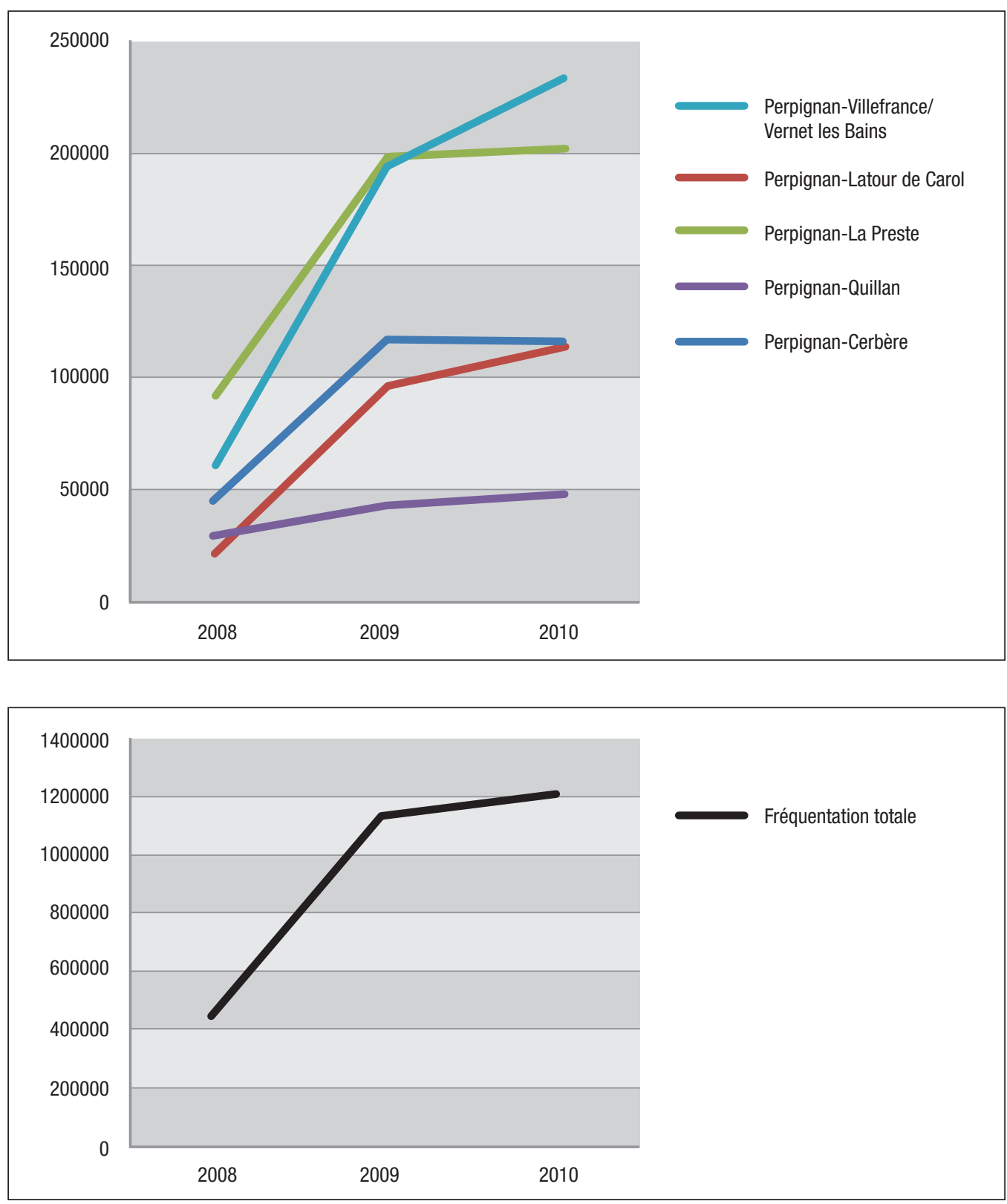

Illustration 2 : Évolution du trafic de voyageurs sur les principales lignes départementales du «Bus à 1 euro » entre 2008 et 2010 (source : Conseil Général des Pyrénées-Orientales (2012)).
Illustration 3 : Évolution du trafic voyageur total sur l'ensemble du réseau du «Bus à 1 euro » entre 2008 et 2010 (source : Conseil Général des Pyrénées-Orientales (2012)).
Déficitaires et jusque-là peu adaptées aux besoins des usagers des transports publics, les lignes des bus départementaux ne jouaient qu'un rôle symbolique dans l'aménagement et la structuration du territoire. L'innovation vint sous l'instigation du Conseil Général des Pyrénées-Orientales du lancement au $1^{\text {er }}$ novembre $2008 \mathrm{du}$ "Bus à 1 euro » pour tous, tout le temps et toute l'année sur l'ensemble du territoire des PyrénéesOrientales. En misant sur une baisse importante et une uniformisation du prix du ticket de transport, le Conseil Général a contribué à faire accroître considérablement le trafic passager (voir illustration 2 et 3), tout en contenant les déficits d'exploitation.

Un an après le lancement du «Bus à un euro", Patrice Rémy (Belmont, 2009), ancien directeur du Pôle transport au
Conseil général, et tout nouveau directeur du Comité départemental du Tourisme des Pyrénées-Orientales, déclarait qu'en 2008, «les recettes des bus s'élevaient à 900000 euros. Ce sera exactement la même ligne de budget cette année. La hausse de la fréquentation a compensé la baisse du prix. Nous avons même réussi à fidéliser les nouveaux usagers. Pour équilibrer les comptes, les dessertes ont été rationalisées, en remplaçant notamment des lignes régulières par des services à la demande pour éviter de faire rouler des bus à vide. En 2012, toutes les communes seront ainsi desservies». La notoriété du «Bus à 1 euro » auprès des touristes se manifeste chaque été par une forte hausse du trafic voyageur. On observe également au niveau des offices de tourisme, une demande croissante et soutenue d'informations relatives aux horaires et trajets de 
l'autobus de la part des vacanciers qui saisissent là l'opportunité de visiter le territoire départemental à moindre coût. Ces nouvelles mobilités spatiales génèrent une dynamique d'ouverture et d'intégration de l'arrière-pays. Elles favorisent de nouvelles solidarités interterritoriales, contribuant ainsi à maintenir le tissus économique et social de l'arrière-pays tout en structurant davantage le territoire départemental.

Dans la foulée du succès du «Bus à 1 euro » des PyrénéesOrientales et des Alpes-Maritimes (un euro également), 29 départements ont choisi cette formule du tarif unique, entre 1,50 et 2 euros.

\section{Mettre la coopération transfrontalière au cœur du développement durable du tourisme}

Au cœur de la vie économique, sociale et culturelle des territoires, un certain type de tourisme facilite les échanges et les rencontres entre acteurs professionnels, permettant ainsi de développer des réseaux sociaux qui constituent la première étape dans la mise en place de projets transfrontaliers de coopération sur des problématiques communément partagées. Appréhendée dans un contexte transfrontalier, la thématique touristique met en jeu des questions relevant à la fois du développement durable, autour de l'entretien et de la valorisation du patrimoine historique, culturel et naturel mais aussi des questions de promotion des territoires comme destinations touristiques intégrées.

Le patrimoine roman ou les paysages ne connaissant pas de frontière, il était important dans un souci de gestion harmonieuse des ressources patrimoniales et de mise en valeur de l'identité catalane de travailler en partenariat avec la Catalogne en s'appuyant sur les fonds européens de cohésion (FEDER, INTERREG) mise en place dans le cadre de la coopération transfrontalière. Cette mise en valeur de l'identité s'est réalisée avec la création du premier Pays d'Art et d'Histoire transfrontalier réunissant les comarques (territoires administratifs historiques en Catalogne) du Vallespir, du côté français, et celles de l'Alt Empordà, de la Garrotxa et du Ripollès, côté espagnol. Dès l'été 2011, plusieurs visites guidées du patrimoine furent ainsi organisées en catalan et en français de part et d'autres de la frontière, permettant ainsi d'inscrire les actions de développement touristique au sein d'un espace transfrontalier culturellement pertinent et homogène, le tout dans une démarche de durabilité. Ces visites guidées se sont poursuivies à l'été 2012 avec une offre similaire à celle de 2011.

\section{Conclusion}

Si l'arrière pays des Pyrénées-Orientales est longtemps resté marginalisé par la fulgurance du développement du littoral départemental, il symbolise aujourd'hui le renouveau d'une destination méditerranéenne qui se construit à présent selon un modèle durable de développement touristique. Protégé de l'impact du tourisme de masse par son isolement géographique et économique, l'arrière-pays représente aujourd'hui un atout essentiel pour la promotion des Pyrénées-Orientales de part la richesse de son patrimoine et la préservation de son cadre naturel. Autour de la mise en réseau des acteurs, de la mise en place d'initiatives en faveur des transports en communs et de la promotion du patrimoine historique et naturel local, le développement durable du tourisme touche les équilibres régionaux et territoriaux et constitue par conséquent un enjeu d'aménagement du territoire. Du tourisme de masse au tourisme durable, le département des Pyrénées-Orientales se construit à présent comme une destination touristique globale qui intègre ses terroirs en valorisant l'ensemble de ses richesses géographiques et historiques. «Penser globalement - agir localement» - une idée simple, bien résumée par une formule désormais célèbre qui rappelle que toute ambition de développement durable au niveau international, doit être suivie d'actions concrètes au niveau local.

\section{Références}

BEETON Sue (2006) Community development through tourism, Victoria, Australia, Landlinks Press. 246 p.

BELMONT, Claude (2009) «Succès pour le bus à 1 euro dans les Pyrénées-Orientales», <http://www.lefigaro.fr/ conjoncture/2009/11/24/04016-20091124ARTFIG00434-succes-pourle-bus-a-1-euro-dans-les-pyrenees-orientales-.php>, consulté en mars 2012.

BERTRAND, Léon (2005) Le tourisme, outil de revitalisation des territoires ruraux et de développement durable?, avant propos, Paris, la documentation française. 288 pages.

CAMPBELL, Lisa M. (1999) «Ecotourism in rural developing communities ", Annals of Tourism Research, vol. 26, n 3, p. 534-553.

CAMPBELL, Lisa M. (2002) «Conservation narratives and the received wisdom of ecotourism: Case studies from Costa-Rica ", International Journal of Sustainable Development, volume 5, pages 300-325.

Conseil Général des Pyrénées-Orientales (2012) Direction des Déplacements et de l'Eco-mobilité, Pôle Voyageurs.

CRONIN, Linda (1990) "A strategy for tourism and sustainable tourism», World Leisure and Recreation, vol. 32, $\mathrm{n}^{\circ} 3, \mathrm{p}$ 12-18.

DREDGE, Dianne et John JENKINS (2003) «Destination place identity and regional tourism policy», Tourism Geographies, vol. 5, $\mathrm{n}^{\circ} 4$, p. 383-407.

GEE, Chuck Yim et Eduardo FAYOS-SOLA (1997) «International tourism: a global perspective», Madrid: World Tourism Organization, Madrid. $406 \mathrm{p}$.

GIRODEAU, Gildas (2012) Département des Pyrénées-Orientales. Carte réalisée pour les fins de cette publication.

HAWKINS, Donald E. et Shaun MANN (2007) «The world bank's role in tourism development ", Annals of Tourism Research, vol.34, n², p. 348-363.

HARDY, Anne et Robert J.S. BEETON (2001) «Sustainable tourism or maintainable tourism: managing resources for more than average outcomes », Journal of Sustainable Tourism, vol. 9, n 3, p. 168-192.

HUNTER, Colin (1995) «On the need to re-conceptualise sustainable tourism development", Journal of Sustainable Tourism, Vol. 3, $\mathrm{N}^{\circ} 3$, pp. 155-165.

HUNTER, Colin (1997) «Sustainable tourism as an adaptive paradigm», Annals of Tourism Research, vol. 24, n ${ }^{\circ}$, p. 850-867.

INSEE - Institut national de la statistique et des études économiques (2012) «Populations légales 2009 des Pyrénées-Orientales», Institut national de la statistique et des études économiques, $<\mathrm{http} / / / \mathrm{www}$. insee.fr/fr/ppp/bases-de-donnees/recensement/populations-legales/ departement.asp?dep=66>, consulté en mars 2012.

JONES, Samantha (2005) «Community-based ecotourism: the significance of social capital», Annals of Tourism Research, vol. 32, n 2, p. 303-324. 
MURPHY, Peter E. (1988) «Community driven tourism planning », Tourism Management, vol. 9, $\mathrm{n}^{\circ}$ 2, p. 96-104.

ODT Pyrénées-Orientales, (2011) «Chiffres clés du tourisme des PO en 2010 », Comité départemental du tourisme Pyrénées-Orientales, $<$ http://observatoire-cdt66.typepad.fr/outils/chiffres_cls/index.html>, consulté en mars 2012 .

OMT - Organisation mondiale du tourisme (2005) «Making tourism more sustainable: a guide for policy makers », Organisation mondiale du tourisme, Madrid.

PEARCE, Douglas G. (1992) Tourist organizations, Harlow: Longman Scientific and Technical, 219 p.

PRIDEAUX, Bruce (2000) «The role of the transport system in destination development ", Tourism Management, vol. 21, n 1, p. 53-63.

RÉSEAU CULTUREL (2012) «Qui sommes-nous?», Réseau culturel Terres Catalanes, <http://www.reseauculturel.com/articles. asp?lng=FR\&id=4875>, consulté en mars 2012.

SHARPLEY Richard et David J. TELFER (sous la direction de) (2002), Tourism and development: concepts and issues, Clevedon, England: Aspects of Tourism series Channel View publications. 408 pages.
SIMPSON, Murray (2008) «Community benefit tourism initiatives - a conceptual oxymoron?», Tourism Management, vol. 29, $\mathrm{n}^{\circ}$ 1, p. 1-18.

Tourisme de Terroir (2012) «Un réseau, une marque, un engagement», Fédération départementale de Tourisme de Terroir, <http://www. tourismedeterroir.fr/Un-reseau-une-marque-un-engagement--5598. phtm?lng=FR>, consulté en mars 2012.

Union Européenne (2007) «Agenda pour un tourisme européen compétitif et durable», Europa - Synthèses de la législation de l’UE, <http:// europa.eu/legislation_summaries/enterprise/industry/110132_fr.htm>, consulté en mars 2012.

UNWTO - United Nations World Tourism Organization (2005) Making tourism more sustainable: A guide for policy makers, Madrid, Spain, UNWTO.

WEARING, Stephen et Matthew McDONALD (2002) «The development of community-based tourism: the relationship between tour operators and development agent as intermediaries in rural and isolated communities », Journal of Sustainable Tourism, Volume 10, n 3, p. 191-206.

\section{APPROCHES MÉTHODOLOGIQUES EN TOURISME • APPEL À TEXTES}

Qu'elle soit qualitative ou quantitative, la recherche implique le recours à des techniques de collecte de données et d'analyses qui ont fait leurs preuves. Il arrive cependant que l'expérience touristique, par sa nature particulière (l'étude de phénomènes ou pratiques où certains acteurs sont en mode opérationnels alors que les prestataires sont en mode repos), nécessite une approche particulière. Considérant la nature multidisciplinaire du tourisme, et l'évolution rapides des expériences offertes, les chercheur(e)s sont ainsi confronté(e)s à des défis particuliers.

Il y a un besoin réel de trouver des solutions adaptées aux problèmes engendrés par la recherche en tourisme et de développer des outils adaptés aux situations particulières du domaine. Il est tout aussi important de jeter un regard critique sur les méthodologies employées jusqu'à présent en recherche touristique.

Téoros est à la recherche de textes consacrés à la méthodologie de recherche en tourisme, soit à propos de nouvelles approches, soit à propos de nouvelles façons de recourir aux méthodes de recherches existantes. Seront considérés les articles développés autour de discussions conceptuelles ou encore sur les applications de diverses méthodes. En plus de ces aspects, votre article peut porter sur:

- de nouvelles méthodes de recherche en tourisme selon les disciplines concernées par le phénomène;

- de nouvelles façons d'employer des méthodes de recherche conventionnelles en tourisme;
- des façons créatives de combiner les méthodes qualitative et quantitative;

- de nouvelles approches pour l'étude des questions contemporaines qui impliquent l'effort de communications entre les disciplines (comme, par exemple, la nature interdisciplinaire croissante des études en tourisme et le développement accéléré des études virtuelles).

Les auteur(e)s doivent faire parvenir un manuscrit rédigé préférablement en français présenté selon les règles de la revue, disponibles à l'url: http://teoros.revues.org/168. Les textes soumis, en format Word, doivent compter environ 7000 à 7500 mots et doivent comprendre un objectif (question) de recherche clairement énoncé ; un descriptif de la méthodologie de recherche employée, et un volet théorique. Une étude de cas peut s'ajouter à ces éléments.

Chaque article doit inclure les nom et prénom de tous les auteurs, leur titre principal et leur affiliation (une seule), leur adresse électronique (courriel) et postale, un résumé de 150 à 200 mots maximum en français ainsi qu'une une liste des mots clés (maximum de 5). II n'y a pas de date limite pour soumettre un texte sur les approches méthodologiques: Téoros les reçoit en tout temps. Les propositions de textes doivent être adressées à la revue: teoros@ uqam.ca. Veuillez inscrire "Méthodologie» dans la ligne de sujet.

Au plaisir de vous lire dans nos pages! 